\title{
Survival of calcium pyrophosphate crystals in stored synovial fluids
}

\author{
Neil W McGill, Angela Swan, Paul A Dieppe
}

\begin{abstract}
Eleven synovial fluids containing calcium pyrophosphate dihydrate (CPPD) were examined repeatedly over an eight week period to assess whether storage conditions and duration influenced the number of crystals present. Aliquots of each fluid were stored at room temperature, $4^{\circ} \mathrm{C}$, and $-70^{\circ} \mathrm{C}$. At $-70^{\circ} \mathrm{C}$ there was no change in crystal count after eight weeks' storage. At room temperature and $4^{\circ} \mathrm{C}$ crystal counts declined slowly over the eight week period, though CPPD crystals were still readily apparent after eight weeks in $10 / 11\left(4^{\circ} \mathrm{C}\right.$ ) and $8 / 11$ (room temperature) fluids. No change in crystal morphology was detected and, apart from one fluid kept at room temperature in which fungal hyphae were noted at six weeks, no new crystals were seen. Calcium pyrophosphate dihydrate crystals in synovial fluid can be maintained for prolonged periods by freezing.
\end{abstract}

Examination of synovial fluid for crystals is a standard component of the assessment of a patient with suspected crystal related arthritis. Little is known, however, about the survival of crystals in stored synovial fluids, though attention has been drawn to the formation of new crystals $^{1}$ and to the dissolution of existing crystals. ${ }^{2}$ Changes in cell and crystal counts in stored synovial fluids have recently been reported. ${ }^{3}$ Five fluids were initially 'laden with crystals of calcium pyrophosphate dihydrate (CPPD)', but by the following day these crystals were difficult to recognise and all had disappeared within 3-8 weeks. Refrigeration at $4^{\circ} \mathrm{C}$ made little difference compared with fluid stored at room temperature.

These findings were not in accord with our experience of synovial fluids containing CPPD stored in a refrigerator over many weeks, and thus we studied prospectively the change in CPPD crystal counts in consecutive synovial fluids which were initially positive for CPPD crystals.

Rheumatology Unit, Bristol Royal Infirmary, Bristol

N W McGill

A Swan

P A Dieppe

Correspondence to:

Dr N W McGill,

Rachel Forster Hospital,

150 Pitt Street,

Redfern,

Redfern,

Australia.

Accepted for publication

18 October 1990

\section{Methods}

Eleven synovial fluids containing CPPD from eight patients were studied. Fluids were collected into plain sterile plastic containers without anticoagulant. The method of examination was identical on each occasion. Fluids were briefly rotamixed, but not centrifuged, then a single drop of synovial fluid was pipetted onto an acid washed glass slide, a cover slip applied, and crystal counts determined by counting the number of CPPD crystals in nine noncontiguous high power fields $(625 \times$ magnification) in a set pattern so as to avoid bias and minimise sampling error. An initial examination was made within 30 minutes of aspiration and then each fluid was divided and put into three plastic sterile screw top containers, which were then stored at room temperature, $4^{\circ} \mathrm{C}$, and $-70^{\circ} \mathrm{C}$ respectively. A few fluids were also stored in heparinised tubes. Repeat crystal counts of the aliquots stored at room temperature and $4^{\circ} \mathrm{C}$ were made at one, two, and six hours, one and two days, one, two, four, six, and eight weeks. Aliquots stored at $-70^{\circ} \mathrm{C}$ were reexamined at eight weeks. The fluids were examined by an observer who was unaware of previous crystal counts obtained for the fluid. The mean coefficient of variation for crystal counts was $0 \cdot 10$.

The observer was also allowed to record a comment on the microscopic appearance of each synovial fluid aliquot.

Statistical significance of difference in crystal counts was assessed with the Wilcoxon matched pairs test (two tailed).

\section{Results}

The morphology of the crystals was typical of CPPD: predominantly, slender rods (length usually $<8 \mu \mathrm{m}$ ), which showed weakly positive birefringence and, less often, rhomboids, which showed stronger positive birefringence. Crystals were often intracellular. No change in morphology or size of the crystals was detected after storage for up to eight weeks.

The table presents the change in CPPD crystal counts for the group of fluids over the eight week storage period. Figure 1 shows the counts for each individual fluid. Crystal counts changed only slowly and in all cases crystals were still readily apparent at two weeks. In 8/11 aliquots stored at room temperature and 10/11

\begin{tabular}{|c|c|c|c|}
\hline \multirow{2}{*}{$\begin{array}{l}\text { Duration of } \\
\text { storage before } \\
\text { crystalcount }\end{array}$} & \multicolumn{3}{|c|}{ Storage conditions } \\
\hline & $\begin{array}{l}\text { Room } \\
\text { temperature }\end{array}$ & $4^{\circ} \mathrm{C}$ & $-70^{\circ} \mathrm{C}$ \\
\hline $\begin{array}{l}\text { Initial count } \\
1 \text { Hour } \\
2 \text { Hours } \\
\text { 6 Hours } \\
\text { 1 Day } \\
\text { 2 Days } \\
\text { 1 Week } \\
\text { 2 Weeks } \\
\text { 4 Weeks } \\
\text { 6 Weeks } \\
8 \text { Weeks }\end{array}$ & $\begin{array}{l}27(14) \\
25(13) \\
27(15) \\
28(19) \\
26(16) \\
25(16) \\
22(12) \\
20(11) \\
13(9) \\
12(8) \\
6(10)\end{array}$ & $\begin{array}{l}27(14) \\
25(13) \\
23(11) \\
26(15) \\
23(15) \\
24(14) \\
21(13) \\
20(11) \\
15(8) \\
14(9) \\
13(10)\end{array}$ & $27(14)$ \\
\hline
\end{tabular}



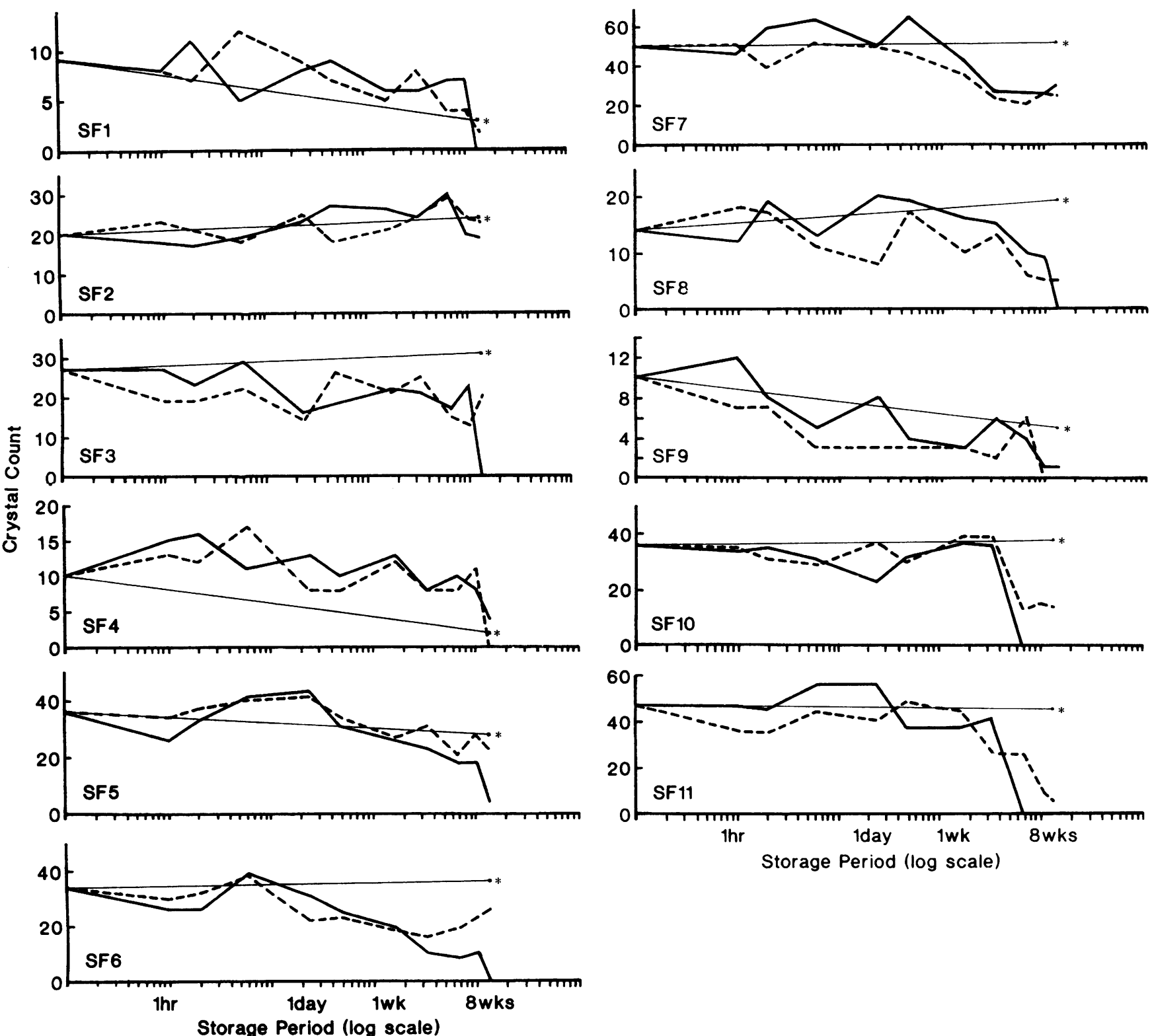

Figure 1 Crystal counts at intervals over eight weeks for each of the 11 synovial fluids $-=$ room temperature $;---=4^{\circ} \mathrm{C} ;-70^{\circ} \mathrm{C} . \mathrm{Note}$ log scale on $x$ (time) axis. In SF 1, SF3, and SF8 (room temperature, eight weeks) crystals were visible in clumps of cells outside the nine fields used for measurement.

Figure 2 Polarised light photomicrograph of synovial fluid after eigth weeks' storage at $-70^{\circ} \mathrm{C}$. Many calcium pyrophosphate dihydrate crystals can be clearly seen.

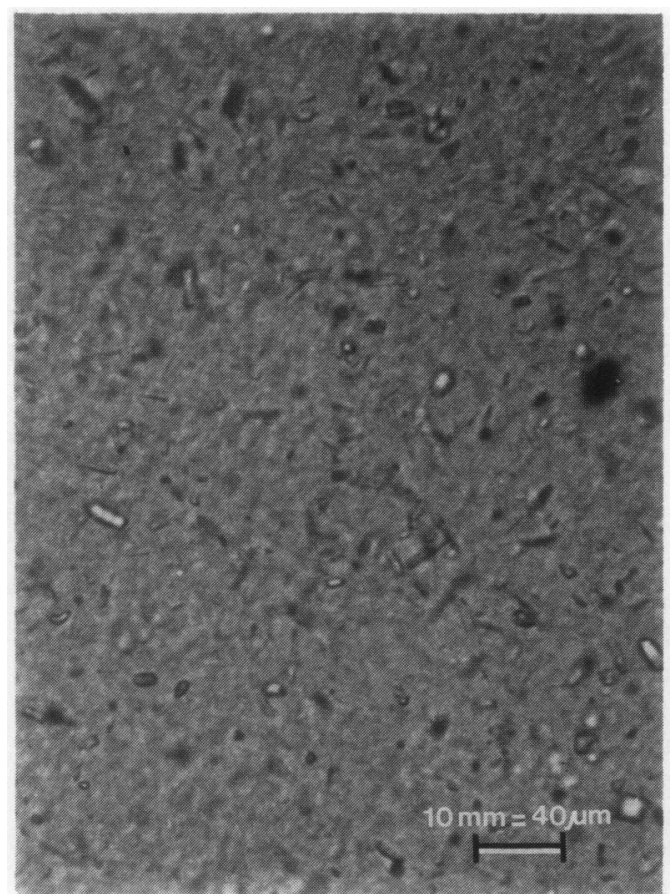

aliquots stored at $4^{\circ} \mathrm{C}$ crystals were still readily apparent at eight weeks. For synovial fluids stored at room temperature and $4^{\circ} \mathrm{C}$ the crystal counts were significantly reduced at eight weeks $(\mathrm{p}=0.001$ and $\mathrm{p}=0.002$ respectively); at one week the difference just failed to reach significance $(p \leqslant 0 \cdot 1)$ for fluids kept at room temperature but achieved significance for fluids kept at $4^{\circ} \mathrm{C}(\mathrm{p}<0.02)$. Aliquots stored at $-70^{\circ} \mathrm{C}$ had no change in crystal counts after eight weeks (p>0.4) (fig 2).

When the comments on the microscopic appearance of each aliquot were reviewed it was found that fluids in which the crystal count had declined substantially were those in which prominent clumping of cells and debris had been recorded. Fluids stored at $-70^{\circ} \mathrm{C}$ did not display cell clumping. Aliquots stored in heparinised containers behaved similarly to those in plain containers.

No brushite crystals were seen. A single aliquot stored at room temperature was noted to contain fungal hyphae and strongly negatively birefringent unidentified crystals at six weeks. 
No crystals other than CPPD were seen in any of the other aliquots at any stage during the study.

\section{Discussion}

Our results clearly show that CPPD crystals in synovial fluid do not rapidly disappear on storage, though crystal counts do tend to reduce after one week when the fluids are maintained at room temperature or in the fridge at $4^{\circ} \mathrm{C}$. Freezing the synovial fluid preserves the CPPD crystal count and prevents clumping of cells and debris. Our findings differ from those of Kerolus et $a l,{ }^{3}$ who found a much more rapid disappearance of CPPD crystals from five synovial fluids.

Why the crystals disappear at all is not clear. The solubility of CPPD crystals is low but pyrophosphatase activity is present in synovial fluid $^{45}$ and this activity might vary in different fluids. Freezing probably reduced both the solubility of the crystals and any pyrophosphatase activity and certainly it reduces the tendency of white cells to clump with storage, all of which helps to maintain the number of CPPD crystals which can be seen. The apparent correlation between the degree of clumping of white cells and debris and the disappearance of the crystals in fluids kept at room temperature or $4^{\circ} \mathrm{C}$ suggests that clumping plays a part in determining apparent crystal numbers. Freezing might increase the risk of new crystals forming during storage, but we found no evidence of this.

We recommend that synovial fluids should be examined promptly after aspiration and note that a failure to do so will result in a misleading cell count ${ }^{3}$ and may allow crystal formation to occur in vitro. ${ }^{1}$ If examination must be delayed, or one wishes to keep the fluid, it should be stored in a freezer in a plain container. Under these conditions the crystal count will remain stable for at least eight weeks and probably much longer.

This study was supported by the Medical Foundation, University of Sydney, and the Arthritis and Rheumatism Council, UK.

1 Dieppe P, Hornby J, Swan A, Hutton C, Preece A Laboratory handling of crystals. Ann Rheum Dis 1983; 42 (suppl):

2 Reardon J A, Scott J T. Resolution of acute gout attacks: a possible mechanism. Ann Rheum Dis 1980; 39: 189.

3 Kerolus G, Clayburne G, Schumacher H R. Is it mandatory to examine synovial fluids promptly after arthrocentesis? Arthritis Rhewo 1989; 32: 271-8.

4 Jacobelli S, Kettlun A M, Sapag-Hagar M. Inorganic pyrophosphatase activity of the synovial fluid. Kinetic and clincal study. Arthritis $R$ heum $1978 ; 21: 447-52$.

5 Caswell A, Guilland-Cumming D F, Hearn P R, McGuire M K B, Russell R G G. Pathogenesis of chondrocalcinosis and pseudogout. Metabolism of inorganic pyrophosphate and production of calcium pyrophosphate dihydrate crystals. Ann Rheum Dis 1983; 42 (suppl): 27-37. 